\title{
The sleeper effect between psychotherapy orientations: a strategic argument of sustainability of treatment effects at follow-up
}

Received 6 September 2016; Accepted 4 October 2016; First published online 28 October 2016

Key words: Randomized controlled trial, comparative psychotherapy outcome research, follow-up, evidence based practice.

Over the past 100 years, claims that specific psychotherapeutic orientations produce more sustainable outcomes, when compared with other orientations or interventions common across psychotherapies, have been pervasive and influential. This kind of differences in relative efficacy over follow-up time periods is referred to as the 'sleeper effect', i.e., the effects of psychotherapy A against psychotherapy B produces larger treatment differences at follow-up than at postassessment (see Fig. 1).

\section{Background}

Psychotherapeutic treatments conducted by trained professionals that are intended to be therapeutic (i.e., bona fide psychotherapy) are shown to be highly effective for treating many mental health conditions. Interestingly, there are marginal to small differences in efficacy between the various bona fide psychotherapy orientations (e.g., cognitive behavioural therapy $v$. Psychodynamic therapy) at post-treatment, so attention has turned towards examining any potential sleeper effects that might favour one treatment over another. The sleeper effect has been formulated in the following ways:

- The depth psychodynamic therapies: Lasting structural personality changes will only be achieved by a profound look into the depths of the mind. In comparison with more behaviourally oriented treatments, insight-oriented psychodynamic therapies work through the subconscious and therefore the effects are more sustainable (Shedler, 2010).

- The carefully controlled evidence-based (mostly cognitive-behavioural) therapies: Disorder-specific evidence-based therapies with close adherence to

*Address for correspondence: Professor C. Flückiger, Ph.D., SwissBPP, Department of Psychology, Division of Psychological Interventions and Psychotherapy, University of Zürich, Binzmühlestrasse 14/04, CH-8050 Zürich, Switzerland.

(Email: christoph.flueckiger@psychologie.uzh.ch) treatment manuals/guidelines under randomised controlled study conditions have been carefully developed and tested. Therefore, their effects will be more sustainable in comparison with less controlled treatments (Tolin, 2010).

- The specificity of additive component designs: Additive intervention components address specific therapeutic aspects, which may result in broader patient competencies and more sustainable treatment effects in comparison with treatments with less systematic focus on the specific ingredient (Bell et al. 2013).

\section{Empirical evidence}

To test the presence of the sleeper effect, the statistical results of randomised controlled psychotherapy trials should indicate a treatment (psychotherapy A v. B) by time (post $v$. follow-up) interaction. In parallel, longitudinal meta-analyses can test these treatment by time interactions in direct treatment comparisons.

We searched the term 'longitudinal meta-analysis' and 'psychotherapy' and 'follow-up' in PsycINFO and MEDLINE (September 30, 2016) and found 18 hits from which three meta-analyses of the direct treatment comparisons provided a statistical test of the sleeper effect (Flückiger et al. 2014, 2015; Kivlighan et al. 2015). In addition, one longitudinal meta-analysis tested the longitudinal course within a treatment (Hanrahan et al. 2013):

- Psychodynamic v. non-dynamic psychotherapies ( $k=20$ primary studies): Findings indicated equivalent efficacy at post-treatment and at follow-up, no sleeper effect (Kivlighan et al. 2015).

- Evidence-based psychotherapy $v$. treatment as usual $(k=15)$ : Small-to-moderate differences in efficacy were found in favour of evidence-based treatments at post-treatment but no extended efficacy at follow-up. The treatment as usual conditions ranged from the absence of minimal mental health treatment to legitimate psychological interventions provided 


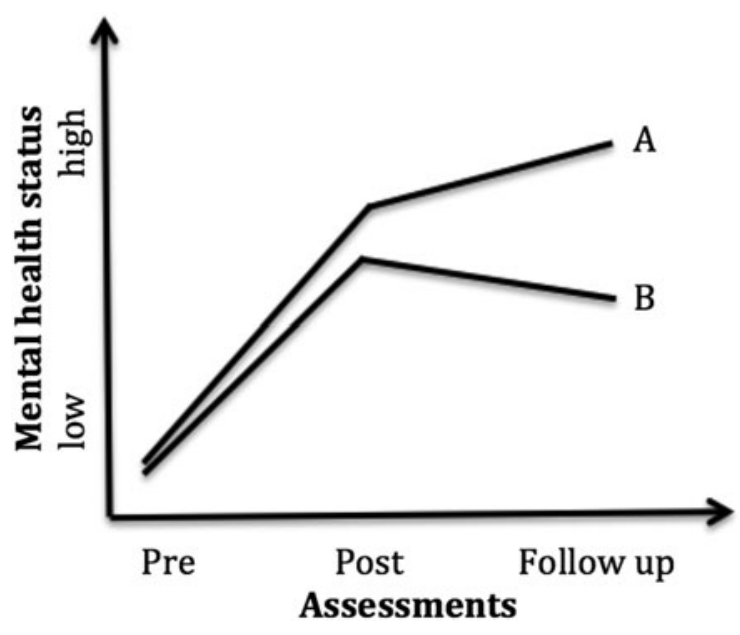

Fig. 1. Example of the sleeper effect: More sustainable outcomes at follow-up in favour of psychotherapy A in comparison with psychotherapy $\mathrm{B}$.

by trained professionals, the latter of which indicated small differences in relative efficacy at posttreatment as well as at follow-up, no sleeper effect (Wampold et al. 2011; Flückiger et al. 2014).

- Additive components $v$. usual components $(k=30)$ : A re-analysis of Bell et al. - meta analysis (2013) found marginal to small differences in efficacy at post-treatment and no extended efficacy at follow-up in favour of additive components (Flückiger et al. 2015). In parallel, the longitudinal course in cognitive-behavioural therapy in generalised anxiety was tested and marginal differences in efficacy were found at post-treatment in favour to treatments with additive components. However, no extended efficacy at follow-up was found, no sleeper effect ( $k=17$, Hanrahan et al. 2013).

\section{Conclusions}

Historically, the argument that some psychotherapy orientations produced more sustainable outcomes at follow-up than others contributed to the split of the psychotherapy community into different camps. The most up-to-date meta-analytic evidence indicates a surprisingly different, yet consistent picture: There is marginal empirical evidence for the presence of a sleeper effect in comparative psychotherapy research. None of the meta-analyses found a growth of relative efficacy at follow-up assessment. While the theoretical arguments for differences in efficacy (depth, carefully controlled, specificity) are reasonable, there is little theoretical reason for why these effects would only notably appear at follow-up assessment points. Clearly, there is variation in the sustainability. However, the attribution of such an effect to a single psychotherapy orientation is not justified based on the evidence. Methodologically, potential confounds such as dropout rates, external events over time and additional treatments may have made it difficult to detect differences in relative efficacy at follow-up (Knekt et al. 2008; Durham et al. 2012).

Nevertheless, the sleeper effect as a lasting theoretical claim has survived over the past 100 years. The sleeper effect might be thought of as a strategic effect in the sense that the dominant and most powerful orientations in this time (earlier psychodynamic therapies, now maybe evidence-based psychotherapies) are 'pulled' to claim sustainability of their own approach; maybe in the hope of further decades of data collection to get an empirical proof of the claim.

\section{Financial support}

None.

\section{Conflict of interest}

None.

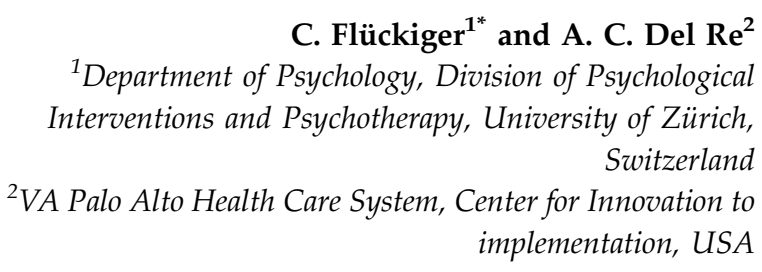

\section{References}

Bell EC, Marcus DK, Goodlad JK (2013). Are the parts as good as the whole? A meta-analysis of component treatment studies. Journal of Consulting and Clinical Psychology 81, 722-736.

Durham RC, Higgins C, Chambers JA, Swan JS, Dow MGT (2012). Long-term outcomes of eight clinical trials of CBT for anxiety disorders: symptom profile of sustained recovery and treatment-resistant groups. Journal of Affective Disorders 136, 875-881.

Flückiger C, Del Re AC, Munder T, Heer S, Wampold BE (2014). Enduring effects of evidence-based psychotherapies in acute depression and anxiety versus treatment as usual at follow-up - a longitudinal meta-analysis. Clinical Psychology Review 34, 367-375.

Flückiger C, Del Re AC, Wampold BE (2015). The sleeper effect: artifact or phenomenon - A brief comment on are the parts as good as the whole? A meta-analysis of component treatment studies (Bell, Marcus \& Goodlad, 2013). Journal of Consulting and Clinical Psychology 83, 438-442.

Hanrahan F, Field AP, Jones FW, Davey CGL (2013). A meta-analysis of cognitive therapy for worry in generalized anxiety disorder (2013). Clinical Psychology Review 33, 120-132. 
Kivlighan DM, Goldberg SB, Abbas M, Pace BT, Yulish NE, Thomas JG, Cullen MM, Flückiger C, Wampold BE

(2015). The enduring effects of psychodynamic treatments vis-à-vis alternative treatments: a multilevel longitudinal meta-analysis. Clinical Psychology Review 40, 1-14.

Knekt P, Lindfors O, Härkänen T, Välikoski M, Virtala E, Laaksonen MA, Virtala E (2008). Randomized trial on the effectiveness of long and short term psychodynamic psychotherapy and solutionfocused therapy on psychiatric symptoms during a 3 year followup. Psychological Medicine 38, 689-703.
Shedler J (2010). The efficacy of psychodynamic psychotherapy. American Psychologist 65, 98-109.

Tolin DF (2010). Is cognitive-behavioral therapy more effective than other therapies? A meta-analytic review. Clinical Psychology Review 30, 710-720.

Wampold BE, Budge S, Laska K, Del Re AC, Baardseth TP, Flückiger C, Minami T, Kivlighan DM, Gunn W (2011). Evidence-based treatments for depression and anxiety versus treatment-as-usual: a meta-analysis of direct comparisons. Clinical Psychology Review 31, 1304-1312. 